\title{
Primary Pulmonary Primitive Neuroectodermal Tumor: A Case Report and Literature Review
}

Guzmán-Casta Jordi' ${ }^{1,10^{*}}$, Riera-Sala Rodrigo ${ }^{1,10}$, Sánchez-Ríos Carla Paola ${ }^{1}$, Hernández-Dehesa Itzel Ariadna ${ }^{4}$, González-Araujo Andrea ${ }^{5}$, Domínguez Ayala Adriana ${ }^{7}$, Correa-Cano Rafael ${ }^{9}$, Elvira-Fabián Karina8, Peña-Mirabal Ericka Sagrario6, López-Vrátný Claudia $^{10}$, Guzmán-Huesca Jorge ${ }^{3}$, Alatorre-Alexander Jorge Arturo ${ }^{1}$, Martínez-Barrera Luis Manuel ${ }^{1}$, Rodríguez-Cid Jerónimo Rafael $^{1}$

${ }^{1}$ Clinic of Thoracic Oncology, Instituto Nacional de Enfermedades Respiratorias, Dr. Ismael Cosío Villegas, Ciudad de México, México

${ }^{2}$ Facultad de Medicina, Universidad Nacional Autónoma de México, Ciudad de México, México

${ }^{3}$ Internal Medicine, Bonita Community Health Center, Florida EE.UU

${ }^{4}$ Department of Radiology, Hospital Ángeles Acoxpa. Ciudad de México, México

${ }^{5}$ Resident of Primary Care, Instituto Mexicano del Seguro Social "IMSS", Guanajuato, México

${ }^{6}$ Department of Pathology, Instituto Nacional de Enfermedades Respiratorias, Dr. Ismael Cosío Villegas, Ciudad de México, México

'Department of Radiooncology, Centro Médico Naxcional 20 de Noviembre "ISSSTE", Ciudad de México, México

${ }^{8}$ Centro Médico Hospital ABC Santa Fé, Ciudad de México, México

${ }^{9}$ Resident of Clinical Oncology, Centro Médico Nacional Siglo XXI "IMSS", Ciudad de México, México

${ }^{10}$ Health Pharma Professional Research, Ciudad de México, México

Correspondence to: Guzmán-Casta Jordi, Clinic of Thoracic Oncology, Instituto Nacional de Enfermedades Respiratorias, Dr. Ismael Cosío Villegas, Ciudad de México, México.

Received date: November 15, 2020; Accepted date: November 25, 2020; Published date: December 2, 2020

Citation: Jordi GC, Rodrigo RS, Carla Paola SR, et al. (2020) Primary Pulmonary Primitive Neuroectodermal Tumor: A Case Report and Literature Review. J Med Res Surg 1(6): pp. 1-3.

Copyright: $@ 2020$ Jordi GC, et al. This is an open-access article distributed under the terms of the Creative Commons Attribution License, which permits unrestricted use, distribution and reproduction in any medium, provided the original author and source are credited.

\section{ABSTRACT}

Primitive Neuroectodermal Tumors (PNET) and Ewing Sarcoma (EWS) belong to the same family of malignant, small, round cell neoplasms of soft tissue or bone origin. Common locations for EWS-PNET include chest wall, pelvis, and extremities. EWSPNETs that arise in the lung parenchyma without chest wall involvement are extremely rare in adults. We report a case of EWS-PNET of the lung in an adult and review the distinct clinical, pathological, and molecular features of these tumors.

\section{Keywords:}

Primitive Neuroectodermal tumor, Lung cancer, Adult.

\section{Introduction}

PNET and EWS belong to the same family of malignant, small, round cell neoplasms of soft tissue or bone origin. EWS-typical PNET's positions include the chest wall, pelvis, and extremities. In adults, EWSPNETs that occur in lung parenchyma without the intervention of the chest wall are exceedingly rare. A case of lung EWS-PNET in an adult is documented and the distinct clinical, pathological, and molecular characteristics of these tumors are investigated.

\section{Clinical Case}

A 77-year-old man with a history of exposure to wood smoke for 60 years, high blood pressure for 4 years on Losartan $50 \mathrm{mg}$ daily. It begins in January 2019 with the progressive presence of pain in the sternum in its lower portion, later with dyspnea on great efforts and weight loss of approximately 10 kilograms. In September 2019 he went to the National Institute of Respiratory Diseases where the approach with a chest tomography with lung injury and tumor infiltration to the thoraco-lumbar spine began, a bronchoscopy was performed with a biopsy of a malignant neoplasm of small and round cells. it is classified as a Neuroectodermal tumor.

He received 30 Gys/10fx palliative radiation therapy to the spine and sternum with pain control and no loss of mobility in the lower extremities. Subsequently, we started systemic treatment with Epirubicin $90 \mathrm{mg} / \mathrm{m} 2$ and Cisplatin $75 \mathrm{mg} / \mathrm{m} 2$ every 3 weeks plus Zoledronic Acid 4 mg monthly.

The patient received 6 cycles of systemic treatment with stable, asymptomatic disease and no evidence of disease progression, starting treatment in November 2019 to March 2020, currently with 8 months free of progression and excellent quality of life.

\section{Radiological Images}

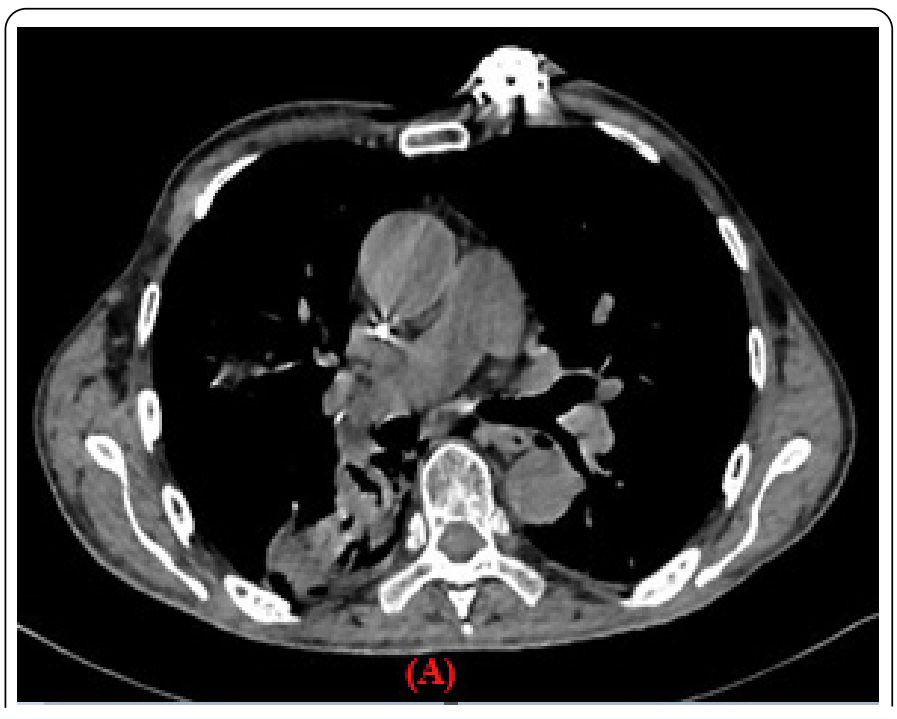



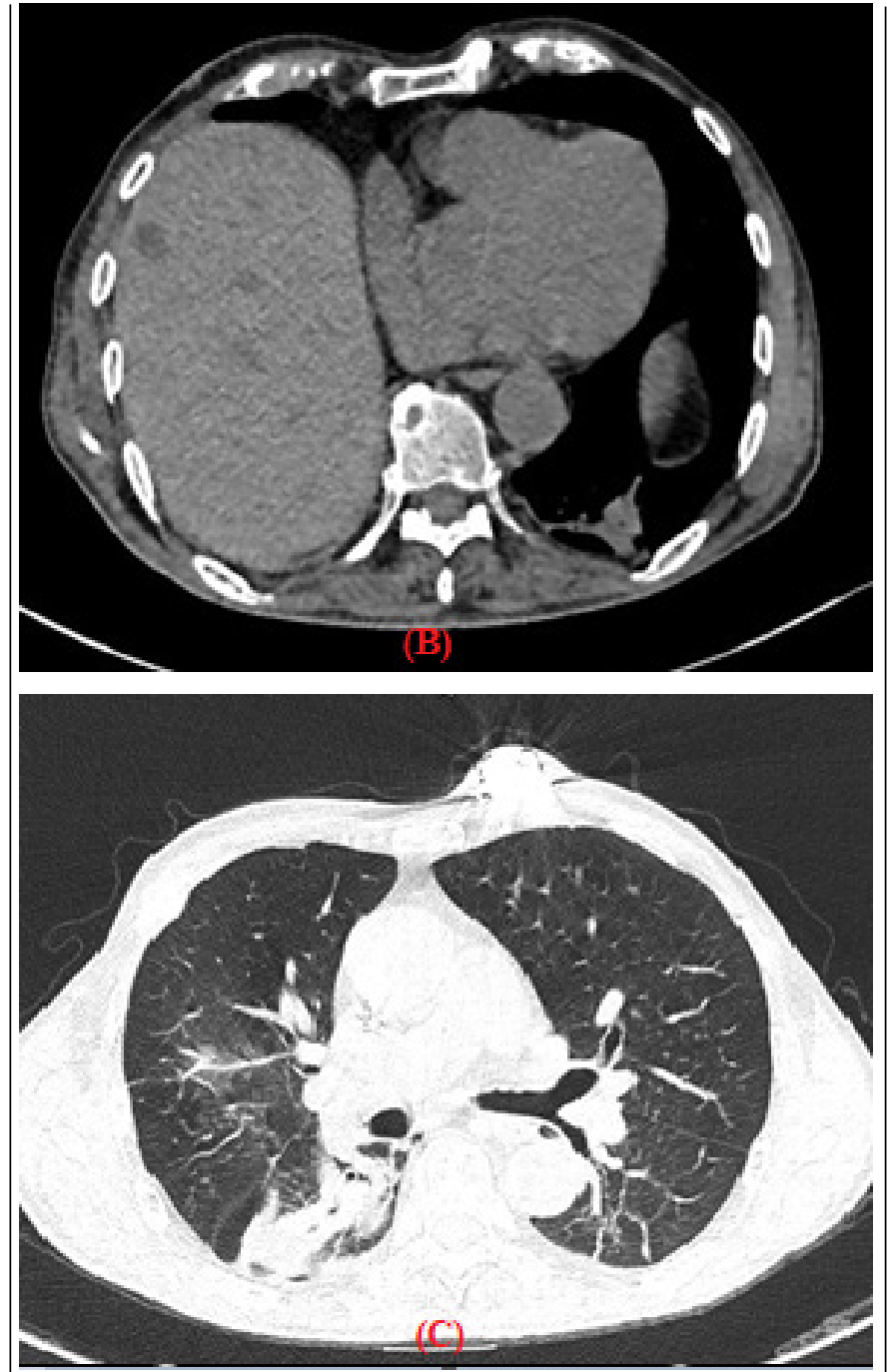

Figure 1: Single-phase computed tomography with window for the mediastinum (A) and (B) and with a pulmonary window (C) in the axial plane, identifying a thin-walled extrapleural tumor located in the posterior right hemithorax, with a density similar to the neighboring muscles, together with the presence of Bilateral pleural thickening and invasion of the lung parenchyma adjacent to it with ground glass halo. The tumor is superimposed and in contact with the costal arches on the left side without being able to identify their destruction. Bilateral as well as subcarinal hilar and perihilar ganglia are observed as evidence of metastatic activity.

\section{Literature Review}

These highly malignant bone and soft tissue tumors are most frequently seen in children and young adults and are histopathologically identified as poorly differentiated "small round cell tumors". Derivation showing varying degrees of neuroectodermal differentiation by ESFT which are tumors of the neural crest [1]. Current evidence indicates that ES and PNET represent a single neoplastic entity, differing only in their degree of neural differentiation. PNETs are the Tumors that demonstrate neural differentiation and Ewing sarcoma are that demonstrate undifferentiated [2].

Due to mass effect, invariably include pain and swelling of the surrounding structures in the site of presentation on Clinical symptoms. Hemoptysis with lung involvement symptoms and signs are site-specific. A combination of imaging tests and information obtained with light microscopy, immunohistochemical staining, and cytogenetic analysis is

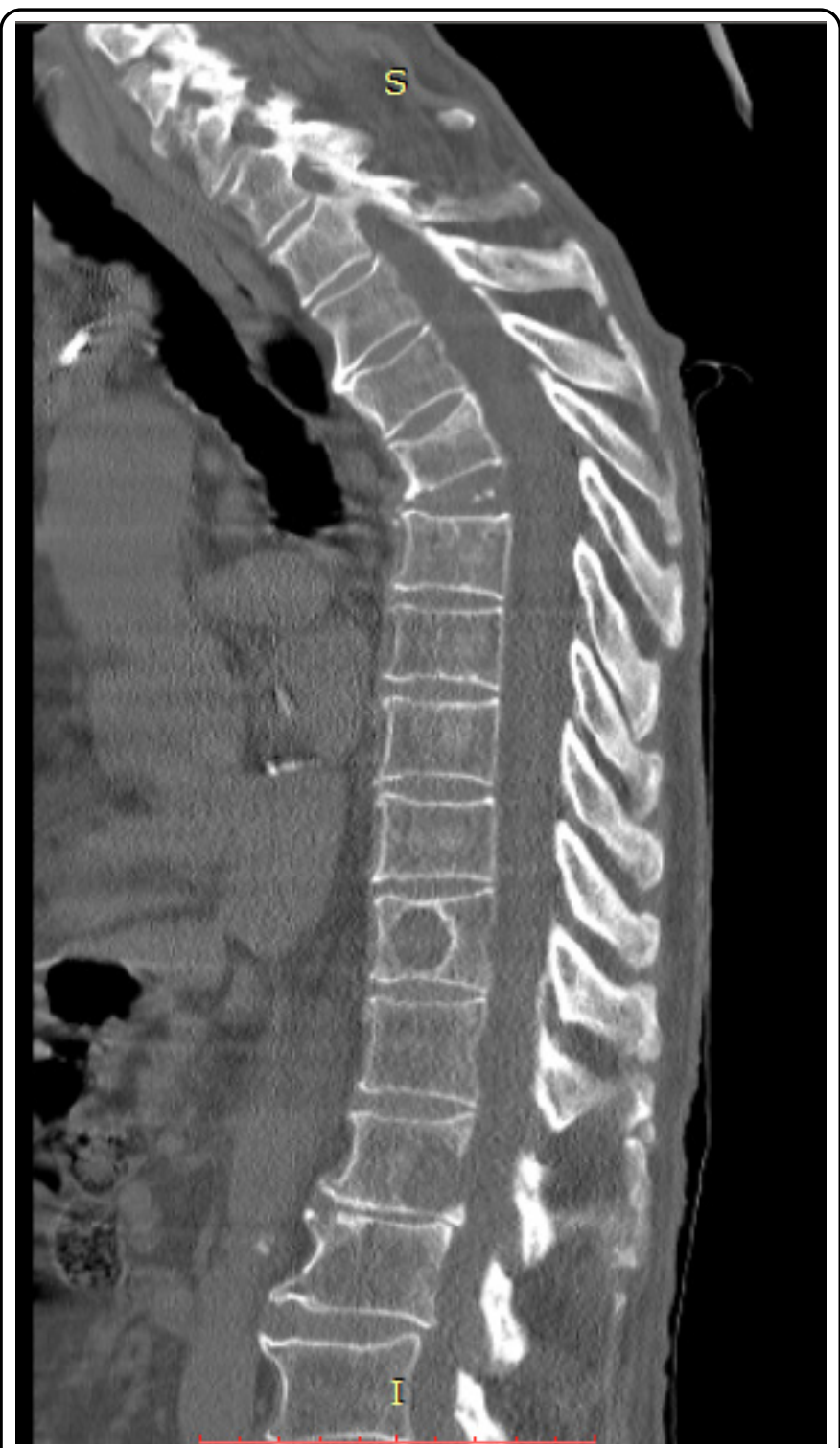

Figure 2: Tomography of the thoracic spine in the sagittal plane bone window. We observed an increase in thoracic kyphosis plus arthritic changes; The presence of lytic-type lesions compatible with tumor activity due to a known primary of at least two vertebral bodies is striking.

\section{Histopathological Images}

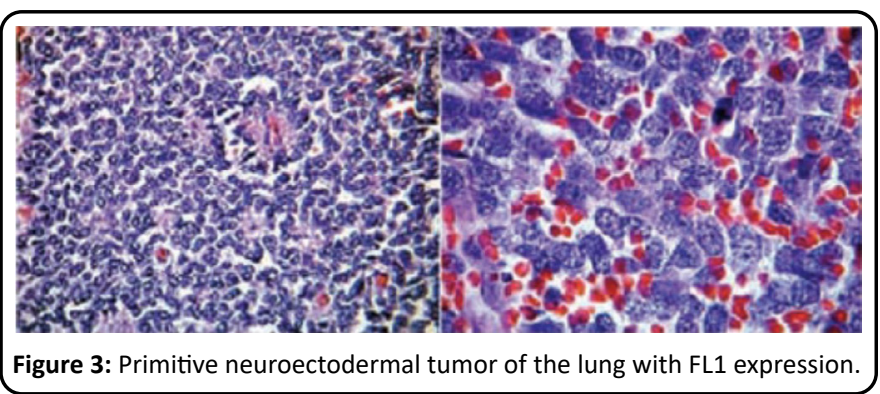

used as a definitive diagnosis of primary pulmonary EWS-PNET. Imaging tests include chest radiographs, CT scans, and MRI to determine the extent of tumor involvement. On CT scans, EWS-PNETs appear as heterogeneous masses, often invading surrounding tissues, including bone. MRI typically reveals a mass isointense to muscle on T1-weighted images, while hyperintense on T2-weighted images. 
Most of the tumors located in the chest show necrosis and multilocular cysts, while some of the tumors appear as homogeneous solid masses [3].

\section{Molecular biology of ESFTs}

ESFTs are distinguished by a particular balanced chromosomal translocation that leads to these tumors being identified as a single entity with a spectrum of phenotypic differentiation. EWS and FLI1 fusion genes are the most common and studied genes. These translocations interrupt specific genes and recombine them to create novel fusion genes that encode tumor-specific proteins [4].

This diagnosis, once difficult for the surgical pathologist, is greatly facilitated now by the availability of antibodies such as HBA-71, 12E7, and 013 that recognize the cell surface antigen defined by the cluster of CD99. Although not specific for PNET or Ewing sarcoma, CD99 is almost always present in these tumors. To exclude lymphoma, carcinoma, or sarcoma, it must be used along with antibodies to vimentin, NSE, neurofilaments, and other indicators defined by the differential diagnosis $[7,8]$.

In lung tumors, the immunohistochemical differential diagnosis is complicated, since CD99 can be found in small cell carcinomas and up to $30 \%$ of carcinoid tumors. Occasionally, PNETs stain with chromogranin and synaptophysin, and aberrant expression of cytokeratin has also been reported. Cytokeratin positive are small cell carcinomas and carcinoid tumors. Thus, a phenotype such as fund in our case, which was CD99, vimentin, NSE, and neurofilament positive and cytokeratin, synaptophysin, and chromogranin negative, is highly suggestive of PNET [8].

\section{Conclusion}

Primitive neuroectodermal tumors are extremely rare, and one of the uncommon sites is the pulmonary location, atypical due to age presentation, knowing that Ewing's Sarcoma is in younger patients and with a high prevalence. Treatment with the most active drugs such as anthracyclines, in this case, Epirubicin and a platinum agent such as Cisplatin with a stable disease response, knowing that the biology of the tumor is difficult to obtain spectacular responses, treatment was also given with palliative radiotherapy and pain control, the use of trimodal therapy with the use of surgery will be evaluated.

\section{References}

1. Scurr M, Judson I (2006) How to treat the Ewing's family of sarcomas in adult patients. Oncologist 11(1): pp. 65-72.

2. Kumar V, Fausto N, Abbas A. Robbins \& Cotran Pathologic Basis of Disease Seventh Edition, 2004.

3. Zhang WD, Xie CM, Mo YX, et al. (2007) CT and MRI features of peripheral primitive neuroectodermal tumor. Ai Zheng 26(1): pp. 643-646.

4. Riggi N, Stamenkovic I (2007) The Biology of Ewing sarcoma. Cancer Lett 254(1): pp. 1-10.

5. Meis-Kindblom JM, Stenman G, Kindblom LG (1996) Differential diagnosis of small round cell tumors. Semin Diagn Pathol 13(3): pp. 213-241.

6. D’Amore ES, Ninfo $V$ (1996) Soft tissue small round cell tumors: morphological parameters. Semin Diagn Pathol 13(3): pp. 184-203.

7. Stevenson AJ, Chatten J, Bertoni F, et al. (1994) CD99 (p30/32MIC2) neuroectodermal/ Ewing's sarcoma antigen as an immunohistochemical marker. Appl Immunohistochem 2(1): pp. 231-240.

8. Hess E, Cohen C, DeRose PB, et al. (1997) Nonspecificity of p30/ 32MIC2 immunolocalization with the 013 monoclonal antibody in the diagnosis of Ewing's sarcoma: application of algorithmic immunohistochemical analysis. Appl Immunohistochem 5(2): pp. 94-103. 